BI-TP 2001/03

November 11, 2018

\title{
Meson Screening Masses at high Temperature in quenched QCD with improved Wilson Quarks
}

\author{
Edwin Laermann and Peter Schmidt \\ Fakultät für Physik, Universität Bielefeld, D-33615 Bielefeld, Germany
}

\begin{abstract}
We report on a lattice investigation of improved quenched Wilson fermions above and below the confinement-deconfinement phase transition. Results on meson screening masses as well as spatial wave functions are presented. Moreover, the meson dispersion relation is studied. Below the critical temperature we do not observe any significant temperature effect while above $T_{c}$ the data are consistent with a leading free quark behavior.
\end{abstract}




\section{Introduction}

An important goal of analytical as well as lattice investigations of QCD at nonvanishing temperature has been to gain more insight into the temperature dependence of hadron properties below and into the nature of hadronic excitations above the transition temperature from the hadronic to the plasma phase of QCD.

When the temperature is raised towards the transition point, approaching (approximate) chiral symmetry restoration and deconfinement is expected to change the properties of hadrons. In particular, the lightest vector mesons and the temperature dependence of their masses and decay widths have received quite some attention because of their possible relevance to the observed enhancement of low mass dileptons in heavy ion collisions [1]. The theoretical predictions of these properties are, however, model dependent, see e.g. [2] for a recent review. In the plasma phase, the effective, temperature dependent coupling constant becomes small at large temperatures. One is thus lead to expect that the plasma consists of a gas of only weakly interacting quarks and gluons. In this case, correlation functions of operators with hadron quantum numbers should be described by the exchange of two or three almost free quarks. On the other hand, there are arguments that even at high temperature the hadronic excitation spectrum might be more complicated because of non-perturbative effects in particular in the chromo-magnetic sector of QCD [3]. Thus, in both temperature regimes ab-initio QCD computations are highly desirable.

Hadronic correlation functions at non-vanishing temperature $T$ have been the subject of lattice investigations for quite a while. Most of these studies are based on the staggered quark formulation 14 -14 while only few so far utilized the Wilson discretization [15 17] and, just recently, domain wall fermions 18]. Moreover, because of the limited extent of the lattice in the Euclidean time direction, $0<t<1 / T$, most of these studies investigated spatial correlation functions and screening masses.

Below the transition temperature, detailed lattice investigations and comparisons of hadron masses at $T=0$ and at $T<T_{c}$ have been carried out in the quenched approximation so far. Analyses in the staggered fermion discretization [8, 12] have covered temperatures between $0.8 T_{c}$ and $0.95 T_{c}$ and did not detect any significant difference between zero-temperature masses and finite-temperature screening masses in the investigated hadron channels. This is also supported by a recent study of spatial and temporal correlation functions of Wilson fermions on anisotropic lattices [17] at $T \simeq 0.93 T_{c}$.

At temperatures above $T_{c}$, the available lattice results on hadronic correlation functions reflect chiral symmetry restoration because the masses [17] and screening masses $40,8,10,13,14$ in the vector $(\rho)$ and axial vector $\left(a_{1}\right)$ channel become degenerate. In the pseudoscalar and scalar channels it is observed that $\pi$ and $f_{0} / \sigma$ become

degenerate at $T_{c}$ [14 while the mass difference between $\pi$ and $a_{0} / \delta$ seems to van- 
ish only at higher temperatures, see in particular [18] for a chiral extrapolation at temperatures closely above $T_{c}$. These findings are in accord with the expected restoration of $S U_{R}\left(N_{F}\right) \times S U_{L}\left(N_{F}\right)$ and indicate that the anomalous $U_{A}(1)$ symmetry is not effectively restored at the critical temperature.

As far as the values are concerned, in the staggered discretization vector and axial vector screening masses are compatible with the prediction of lowest order perturbation theory i.e. the propagation of (almost) free quarks. Scalar and pseudoscalar channels, however, show substantial deviations from this expectation, at least in the temperature interval between $T_{c}$ and $2 T_{c}$. One might argue that this observation indicates the existence of (pseudo) scalar bound states. However, the lowest mass hadron is unlikely to change from a mesonic state to a quark-like quasi-particle at a non-critical temperature. In addition, studies which apply different boundary conditions [11] suggest that the spectral function is dominated by a two quark cut. For Wilson quarks it is observed [17] that already at a temperature of about $1.5 T_{c}$ the pseudoscalar and vector screening masses are very close to each other. There it also is found that a near degeneracy holds true for the masses. Depending on the source operator utilized, the pseudoscalar is sometimes even heavier than the vector meson. While this behavior can at least qualitatively be explained by the propagation of (almost) free quarks, 17 provides conflicting evidence since their study of the wave functions, on the other hand, indicates the presence of bound states.

In addition to these observables, also spatial wave functions have been analyzed [19]. Here one has found a similar behavior as at zero temperature. The observed exponential decay has then been taken as to suggest that the relevant hadronic excitations are bound states also in the plasma phase, at least at temperatures just above $T_{c}$. According to [20], this behavior could, however, also be explained by the fact that the dimensionally reduced, 3-D effective theory and correspondingly spatial Wilson loops in $3+1$ dimensions show confinement [21 23]. Solving a two-dimensional Schrödinger equation with a potential which includes a temperature dependent (spatial) string tension leads to exponentially decreasing spatial wavefunctions. The corresponding effect on the screening masses [24] would be an $\mathcal{O}(10 \%)$ correction at the investigated temperatures 23] which so far could not yet be checked quantitatively.

None of the lattice investigations of hadronic masses at non-vanishing temperatures has attempted to carry out the continuum limit. Most of the mentioned analyses are based on the staggered discretization. A straightforward computation in the Wilson formulation of lattice QCD and a comparison of the results with the staggered ones would thus help to gain an idea about the discretization effects. This is the main goal of this paper.

Since we are extracting screening masses and Lorentz invariance is lost at finite $T$ due to the heat bath, we have also computed spatial correlation functions projected onto some non-vanishing momenta and on the lowest non-vanishing bosonic Matsubara frequency. The purpose of this attempt is to test for a sizeable difference between 
spatial and temporal momentum contributions to the vacuum polarization tensor.

As a by-product of the attempt to construct meson operators with good overlap to the groundstate we also were able to obtain information about the Bethe-Salpeter amplitudes of the investigated mesons, the pion and the $\rho$.

The paper is organized as follows: in the next section we present some details of the simulation. This is followed by the presentation and discussion of the obtained screening masses in the pion and the $\rho$ channel, both below and above the deconfinement transition. In section 4 we test the dispersion relation at non-vanishing temperature. Section 5 contains our estimates of the wave functions.

\section{The simulation}

The results to be presented here are based on gauge field configurations which have been generated with the standard Wilson gluon action. We used a pseudo-heatbath algorithm [26] with FHKP updating [27] in the $S U(2)$ subgroups. Each heatbath iteration is supplemented by 4 overrelaxation steps [28]. We have simulated at three values of the bare coupling, $\beta=6 / g^{2}=6.0,6.2$ and 6.4 . At these $\beta$ values the lattice spacing has been determined from quite a variety of observables. Depending on the quantity, the results spread over a range of about $10 \%$ of the central value, however, within the error bars, agreement is observed. In order to obtain the physical temperature of the lattices in units of the critical one, for definiteness we have consistently chosen to set the scale by the string tension [29], $T / T_{c}=(T / \sqrt{\sigma}) \cdot\left(\sqrt{\sigma} / T_{c}\right)$, taking $T_{c} / \sqrt{\sigma}$ from [30]. The temporal extent of the finite temperature lattices of $N_{\tau}=8$ then corresponds to physical temperatures of $T=0.93(1) T_{c}, 1.23(1) T_{c}$ and $1.63(2) T_{c}$ at $\beta=6.0,6.2$ and 6.4 respectively. The spatial volume of the lattices was chosen as $24^{3}$ and $32^{3}$ at $\beta=6.0,24^{3}$ at $\beta=6.2$ and $24^{2} \times 64$ at $\beta=6.4$. In addition to the finite temperature simulations we have carried out runs at zero temperature on lattices of size $16^{3} \times 32$ at $\beta=6.0$ and $24^{3} \times 48$ at 6.2 mainly in order to supplement the available literature data on meson masses by results at quark masses in the range between the strange and the charm quark mass. We have generated between 50,000 and 80,000 gauge field configurations and have analyzed configurations separated by 500 or 2000 sweeps (see Table 1). Autocorrelations have been checked to be negligible.

For the fermion part of the action we used the $\mathcal{O}(a)$ Symanzik-improved Sheikholeslami-Wohlert action 25] with a tree level clover coefficient of $c_{s w}=1.0$. The inversion of the Dirac matrix was carried out by means of an overrelaxed MR algorithm 31] at moderate to large quark mass values and by the BiCGStabI [32 for light quarks. In both cases an even-odd partitioning was employed. We encountered only very few moderately exceptional configurations. Including or omitting those did not modify the expectation values. With the same combination of actions as in the present study, 
the (latest) values of the critical hopping parameter at zero temperature defined by the vanishing of the pion mass have been determined as $\kappa_{c}=0.14556(6)$ 33 and $\kappa_{c}=0.14549(2)$ 34 at $\beta=6.0, \kappa_{c}=0.14315(2)$ [35 and $\kappa_{c}=0.14315(1)$ 34 at $\beta=6.2$ as well as $\kappa_{c}=0.14143(3)$ [34 at $\beta=6.4$. These numbers may be used to convert the various values of the hopping parameter into an estimate of the corresponding bare quark masses by means of

$$
m_{q} a=\ln \left[1+\frac{1}{2}\left(\frac{1}{\kappa}-\frac{1}{\kappa_{c}}\right)\right]
$$

This has been used also in Table 11 where our run parameters are summarized.

\begin{tabular}{|c|c|c|c|c|c|}
\hline \multicolumn{6}{|c|}{ Simulation Parameters } \\
\hline$\beta \quad a[\mathrm{fm}]$ & Lattice & $T / T_{c}$ & statistics & $\kappa$ & $m_{q}[\mathrm{MeV}]$ \\
\hline \multirow[t]{10}{*}{$\begin{array}{ll}6.0 & 0.105\end{array}$} & $16^{3} \times 32$ & 0 & $\mathcal{O}(100)$ & 0.141 & 200 \\
\hline & & & & 0.130 & 750 \\
\hline & & & & 0.128 & 800 \\
\hline & $24^{3} \times 8$ & 0.93 & $\mathcal{O}(100)$ & 0.141 & 200 \\
\hline & & & & 0.130 & 750 \\
\hline & & & & 0.128 & 800 \\
\hline & $32^{3} \times 8$ & 0.93 & $\mathcal{O}(40)$ & 0.145 & 30 \\
\hline & & & & 0.1445 & 50 \\
\hline & & & & 0.144 & 80 \\
\hline & & & & 0.141 & 200 \\
\hline \multirow[t]{8}{*}{$\begin{array}{ll}6.2 & 0.077\end{array}$} & $24^{3} \times 48$ & 0 & $\mathcal{O}(100)$ & 0.141 & 150 \\
\hline & & & & 0.130 & 800 \\
\hline & & & & 0.128 & 950 \\
\hline & $24^{3} \times 8$ & 1.23 & $\mathcal{O}(30)$ & 0.1428 & 20 \\
\hline & & & & 0.14232 & 60 \\
\hline & & & & 0.14151 & 100 \\
\hline & & & & 0.136 & 450 \\
\hline & & & & 0.128 & 950 \\
\hline \multirow[t]{5}{*}{$\begin{array}{ll}6.4 & 0.058\end{array}$} & $24^{2} \times 64 \times 8$ & 1.63 & $\mathcal{O}(100)$ & 0.1409 & 50 \\
\hline & & & & 0.1406 & 80 \\
\hline & & & & 0.1403 & 110 \\
\hline & & & & 0.14 & 140 \\
\hline & & & & 0.13 & 1100 \\
\hline
\end{tabular}

Table 1: A summary of our run parameters. The physical value of the quark masses has been estimated by using eq. (11) together with the physical value of the lattice spacing as obtained from string tension determinations. For definiteness, $\sqrt{\sigma}$ has been taken as $420 \mathrm{MeV}$.

From the computed quark propagators we constructed correlation functions of op- 
erators with the quantum numbers of the pseudoscalar and the vector meson. To improve the projection onto the lowest energy state, a gauge invariant extended operator was used on the sink site [36]:

$$
\mathcal{M}_{R}(x)=\sum_{ \pm \vec{e}} \bar{\Psi}_{\alpha}^{i}(x) \mathcal{U}^{i, j}(x \rightarrow x+R \vec{e}) \Gamma_{\alpha, \beta} \Psi_{\beta}^{j}(x+R \vec{e})
$$

Here, $\Psi$ and $\bar{\Psi}$ are a quark and an antiquark field separated by a distance $R, i, j$ denote color indices and $\alpha, \beta$ are spinor ones. Both indices are to be summed over. The quantum numbers are selected by choosing $\Gamma=\gamma_{5}$ for the pseudoscalar and $\Gamma=\gamma_{\mu}$ for the vector channel respectively. In the vector channel we have averaged over the polarizations $\mu$ perpendicular to the correlation direction. The explicit sum in eq. (2) is over all unit vectors $\vec{e}$ perpendicular to the correlation direction. This extended operator is made gauge invariant by introducing the color parallel transporter $\mathcal{U}$ from $x$ to $x+R \vec{e}$. In order to further improve the projection and in an attempt to resemble the gluon cloud [36] the parallel transporter is built from smeared link fields. At the source site a strictly local operator $(R=0)$ was put on the lattice.

The most general correlation function $C_{R}(\vec{p}, t)$ is thus obtained as

$$
\begin{aligned}
C_{R}(\vec{p}, t) & =\sum_{\vec{x}} e^{-i \vec{p} \vec{x}}\left\langle P_{R}(\vec{x}, t) P^{\dagger}(\overrightarrow{0}, 0)\right\rangle \\
& =<0\left|P_{R}\right| P(\vec{p})><0|P| P(\vec{p})>^{*}\left\{e^{-E_{P}(\vec{p}) t}+e^{-E_{P}(\vec{p})\left(N_{\tau}-t\right)}\right\}+\cdots
\end{aligned}
$$

where in this particular example the pseudoscalar correlation in the temporal direction was chosen. In eq. (3), the exponential fall-off is given by the energy $E_{P}(\vec{p})$ of the state $\mid P(\vec{p})>$ at momentum $\vec{p}$. The dots indicate contributions from excited states with the right quantum numbers. At non-vanishing temperature, because of the limited extent of the lattices in the temporal direction, $t \leq 1 / T$, we computed spatial correlation functions in the $z$ direction, $C_{R}(\overrightarrow{\tilde{p}}, z)$, where $\overrightarrow{\tilde{p}}$ denote the momentum components perpendicular to the $z$ direction, $\overrightarrow{\tilde{p}}=\left(p_{x}, p_{y}, p_{t}\right)$.

The improvement procedure described above leaves quite some freedom in choosing optimal parameters. As for the distance $R$ between quark and antiquark, on each configuration we have computed the correlation functions for a variety of different $R$ values. This allowed us to find the optimal separation for each lattice spacing and temperature individually. An example of how the separation $R$ changes the projection to the lowest mass state is shown in Figure 1. Here we plot the effective mass, defined as $M^{\text {eff }}(t)=\ln \left\{C_{R}(t) / C_{R}(t+1)\right\}$ for $\vec{p}=\overrightarrow{0}$, of the pseudoscalar as a function of $t$ for various $R$ values. The data has been obtained on the zero temperature lattice at $\beta=6.2$ at $\kappa=0.141$. The plot illustrates that the contribution of excited states becomes considerably smaller when $R$ is raised from 1 to 5 lattice spacings in this example. The data flattens off and reaches a plateau at smaller time separations between source and sink. This allows to extract the lowest mass much more reliably. At $R \geq 7$ the large $t$ limit is approached from below as a single term contributing to 
eq. (3) is not positive-definite. At the rightmost data point in the figure the effective mass drops slightly because the periodicity of the lattice is being felt by the correlation function. When $\beta$ is varied we observe that the optimal distance in lattice units approximately scales with the lattice spacing i.e. stays constant in physical units.

Contrary to the strong $R$ dependence of the effective masses which appears to be physical, details of the gauge field smearing procedure do not seem to matter so much. We have adopted the APE prescription [37] with a weight of 2 for the link term and 1 for the contribution of the staples. As noted also in [36], the precise value of the ratio is not too important, contrary to the case of Wilson loops. The smeared links were projected back to $S U(3)$ elements. In test runs it turned out that varying the number of smearing iterations between 4 and 12 does not have a big impact on the length of the plateau in effective mass plots, see Figure 11. Moreover, between the $\beta$ values analyzed we did not observe significant differences.

\section{Masses}

In this section we present our results on the masses. These were obtained from correlation functions $C_{R}(\vec{p}=0, t)$, eq. (3), at zero momentum in which case the exponential fall-off is given by the mass, $E_{H}(\vec{p}=0)=M_{H}$. For spatial correlation functions the exponential fall-off defines the screening mass.

In order to obtain an estimate of the mass of the lowest state contributing to a

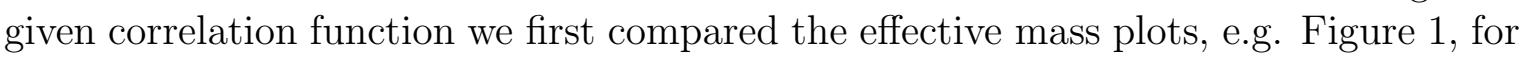
various quark pair distances $R$ in search of the optimal $R$ value with regard to the onset and stability of the plateau. Subsequently, at the chosen $R$ value fits over intervals $\left[t_{\text {min }}, N_{\tau}-t_{\text {min }}\right]$ with varying $t_{\text {min }}$ (similarly for the spatial correlations at $T \neq 0$ ) were performed, again checking for stability of the mass value. Likewise, we symmetrized the correlation functions around midpoint, $N_{\tau} / 2$ and $N_{\sigma} / 2$ respectively, and carried out fits including the full covariance matrix. Again, the minimum separation from the source was varied. The results quoted, mass values as well as errors, are obtained from the latter fits, selecting the fit interval by the best $\chi^{2}$ value. The errors given include an estimate of the systematic error as suggested by a remaining dependence of the mass on $R$ and $t_{\min }$. Finally, also two-state fits were applied to correlation functions including data at next-to-optimal $R$ values and smaller separations from the source in order to further check for consistency.

The results for the ground state masses in the pseudoscalar and the vector channel at the temperatures investigated are summarized in Tables 2 to 1 and are compared with the available zero temperature data in Figures 2 and 3 . In the figures we have 

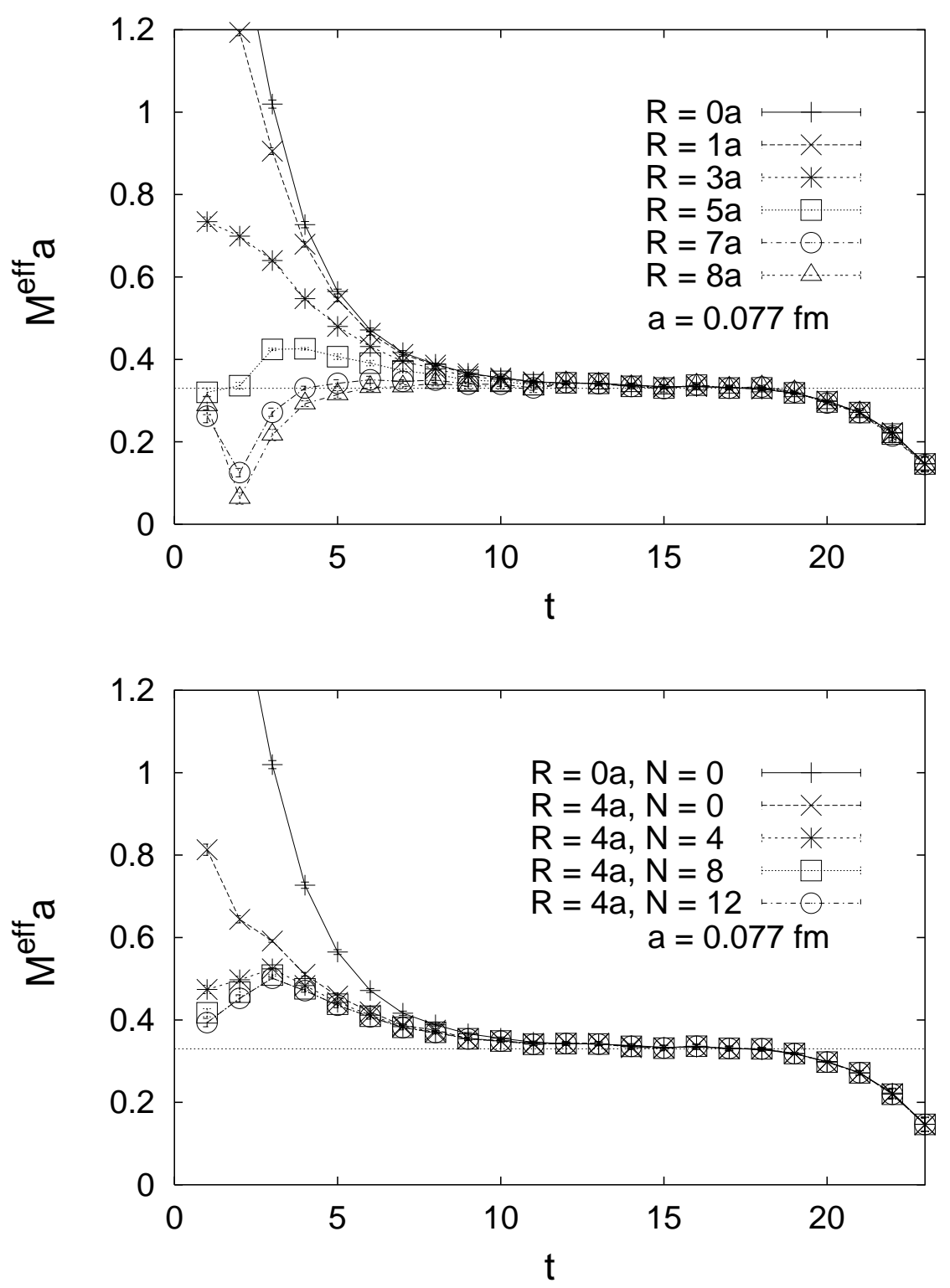

Figure 1: The effective mass $M^{\mathrm{eff}}(t)$ as a function of $t$. The example shows the pseudoscalar channel at zero temperature at $\beta=6.2$ and $\kappa=0.141$. The upper figure demonstrates the effect of varying the distance $R$ between quark and antiquark while the number of smearing iterations was fixed to 4 in this example. In the lower plot, $R$ is fixed at 4 and the number of smearing iterations is varied between 0 and 12 .

plotted the so-called pole mass

$$
m_{H}=2 \sinh \left(\frac{M_{H}}{2}\right)
$$


Eq. (14) arises from a lattice meson action with a nearest neighbor symmetric difference discretization which appears to be favored by studies of lattice dispersion relations [34, 38]. Using $m_{H}$ instead of the coefficient $M_{H}$ of the exponential fall-off partly corrects for $\mathcal{O}(\mathrm{am})$ lattice artefacts. We observe that $m_{V}$ for the vectormeson at zero temperature, unlike $M_{V}$, is strikingly linear in the quark mass up to values in the vicinity of the charm quark mass. For small meson masses the difference between $m_{H}$ and $M_{H}$ is of course negligible.

Figure 2 summarizes the results at $\beta=6.0$ which on the high temperature lattice corresponds to a temperature of $0.93 T_{c}$. The upper part of the figure shows $m_{V}$ and $m_{P}^{2}$ at small quark mass values while the lower part covers the entire quark mass range explored. The meson masses are plotted as function of $m_{q}$ where the quark mass is obtained from eq. (1) with $\kappa_{c}(T=0)$ determined from a linear fit in $m_{q}$ to the combined zero temperature literature data on $m_{P}^{2}$ [33, 34] up to quark mass values $m_{q} a<0.08$. As can be seen from the lowest lying line drawn in the upper part of Figure 2, the linear fit works well and deviations begin to emerge at $m_{q} a \simeq 0.1$. Our result for the critical kappa value is $\kappa_{c}(T=0)=0.14542(2)$ which deviates marginally from the values quoted in [33, 34, 39] because we have chosen a fit procedure slightly different from the one adopted there. Note however, that our value is well within the spread of the quoted $\kappa_{c}$ values. Moreover, the upper part of Figure 2 shows that the finite temperature pion (screening) mass retains a small but non-vanishing value at $\kappa_{c}(T=0)$. A fit to the $m_{P}^{2}$ data at quark mass values up to 0.08 of the form $c+s m_{q}$, shown as the second lowest dotted line, leads to an intercept $c=0.006(3)$ at $m_{q}=0$. The slope $s=3.07(8)$ is a little larger than the value of $s=2.87(3)$ obtained from the equivalent fit to the $T=0$ data. Alternatively, one can perform a fit with a temperature dependent $\kappa_{c}$ leading to a slightly different value, $\kappa_{c}(T)=0.14550(4)$.

For the vectormeson masses, when plotted as a function of the zero temperature quark mass, we find that, as a whole, they apparently tend to be somewhat larger than the zero temperature values. In both cases, fits linear in the quark mass work well and we obtain $m_{V}=0.42(2)$ at $T \simeq 0.93 T_{c}$ as opposed to $0.38(1)$ at $T=0$ in the chiral limit. This is shown as the appropriate lines in the upper part of Figure 2 . At least part of this difference could, however, be absorbed into the shift in $\kappa_{c}$ mentioned above.

In the lower part of Figure $2 m_{V}$ and $m_{P}$ are shown linearly over the entire quark mass range explored. At zero temperature, the vectormeson mass is linear in $m_{q}$ over the entire range. A fit ansatz including a term quadratic in the quark mass returns a value of 0.05(19) for its coefficient which is compatible with 0 . The intercept is obtained as 0.381(6). Within errors this intercept is in agreement with the result of a linear fit, $m_{V}=0.379(2)+2.588(5) m_{q}$ which is shown in the figure. Note, that these numbers are in agreement with the results for the intercept obtained from the fit to the small quark mass data only. The mass of the pseudoscalar shows a behavior proportional to $\sqrt{m_{q}}$ at small quark masses, as expected from chiral perturbation 


\begin{tabular}{|c|c|c|c|}
\hline \multicolumn{4}{|c|}{$\mathbf{1 6}^{\mathbf{3}} \times \mathbf{3 2}, \boldsymbol{\beta}=\mathbf{6 . 0 ,} \boldsymbol{T}=\mathbf{0}$} \\
\hline$\kappa_{1}$ & $\kappa_{2}$ & $M_{P}$ & $M_{V}$ \\
\hline 0.1280 & 0.1280 & $1.259(3)$ & $1.284(4)$ \\
0.1300 & 0.1300 & $1.162(3)$ & $1.191(3)$ \\
0.1410 & 0.1410 & $0.559(3)$ & $0.630(4)$ \\
\hline
\end{tabular}

\begin{tabular}{|c|c|c|c|}
\hline \multicolumn{4}{|c|}{$\mathbf{2 4}^{\mathbf{3}} \times \mathbf{8}, \boldsymbol{\beta}=\mathbf{6 . 0}, \boldsymbol{T}=\mathbf{0 . 9 3} \boldsymbol{T}_{\boldsymbol{c}}$} \\
\hline$\kappa_{1}$ & $\kappa_{2}$ & $M_{P}$ & $M_{V}$ \\
\hline 0.1280 & 0.1280 & $1.262(7)$ & $1.29(1)$ \\
0.1300 & 0.1300 & $1.168(6)$ & $1.197(8)$ \\
0.1410 & 0.1410 & $0.557(8)$ & $0.64(1)$ \\
\hline
\end{tabular}

\begin{tabular}{|c|c|l|l|}
\hline \multicolumn{4}{|c|}{$32^{3} \times \mathbf{8}, \boldsymbol{\beta}=\mathbf{6 . 0}, \boldsymbol{T}=\mathbf{0 . 9 3} \boldsymbol{T}_{\boldsymbol{c}}$} \\
\hline$\kappa_{1}$ & $\kappa_{2}$ & $M_{P}$ & $M_{V}$ \\
\hline \multirow{4}{*}{0.1410} & 0.14100 & $0.559(5)$ & $0.64(1)$ \\
& 0.14400 & $0.463(6)$ & $0.59(1)$ \\
& 0.14450 & $0.443(5)$ & $0.57(1)$ \\
& 0.14500 & $0.42(1)$ & $0.54(1)$ \\
\hline \hline \multirow{3}{*}{0.1440} & 0.14400 & $0.323(5)$ & $0.51(2)$ \\
& 0.14450 & $0.300(1)$ & $0.50(2)$ \\
& 0.14500 & $0.27(1)$ & $0.47(2)$ \\
\hline \hline \multirow{2}{*}{0.1445} & 0.14450 & $0.273(7)$ & $0.46(2)$ \\
& 0.14500 & $0.22(1)$ & $0.44(3)$ \\
\hline \hline 0.1450 & 0.14500 & $0.20(1)$ & $0.44(2)$ \\
\hline
\end{tabular}

Table 2: Meson masses $M_{H}$ of the pseudoscalar and vector meson at $\beta=6.0$, $T=0$ on a $16^{3} \times 32$ lattice and $T=0.93 T_{c}$ on $24^{3} \times 8$ and $32^{3} \times 8$ lattices.

\begin{tabular}{|c|c|c|c|}
\hline \multicolumn{4}{|c|}{$\mathbf{4}^{\mathbf{3}} \times \mathbf{4 8}, \boldsymbol{\beta}=\mathbf{6 . 2}, \boldsymbol{T}=\mathbf{0}$} \\
\hline$\kappa_{1}$ & $\kappa_{2}$ & $M_{P}$ & $M_{V}$ \\
\hline 0.1280 & 0.1280 & $1.124(2)$ & $1.141(2)$ \\
0.1300 & 0.1300 & $1.018(2)$ & $1.040(2)$ \\
0.1410 & 0.1410 & $0.333(2)$ & $0.420(4)$ \\
\hline \multicolumn{4}{|c|}{$\mathbf{3 4 ^ { 3 }} \times \mathbf{8 ,} \boldsymbol{\beta} \mathbf{6 . 2 ,} \boldsymbol{T}=\mathbf{1 . 2 3 \boldsymbol { T } _ { \boldsymbol { c } }}$} \\
\hline$\kappa_{1}$ & $\kappa_{2}$ & $M_{P}$ & $M_{V}$ \\
\hline 0.1280 & 0.1280 & $1.110(4)$ & $1.110(5)$ \\
0.1300 & 0.1300 & $0.995(5)$ & $1.011(4)$ \\
0.1410 & 0.1410 & $0.350(4)$ & $0.410(8)$ \\
\hline
\end{tabular}

\begin{tabular}{|c|c|c|c|}
\hline \multicolumn{4}{|c|}{$\mathbf{2 4}^{\mathbf{3}} \times \mathbf{8}, \boldsymbol{\beta}=\mathbf{6 . 2}, \boldsymbol{T}=\mathbf{1 . 2 3} \boldsymbol{T}_{\boldsymbol{c}}$} \\
\hline$\kappa_{1}$ & $\kappa_{2}$ & $M_{P}$ & $M_{V}$ \\
\hline & 0.12800 & $1.20(2)$ & $1.23(2)$ \\
& 0.13600 & $1.04(1)$ & $1.07(1)$ \\
0.12800 & 0.14151 & $0.96(1)$ & $1.01(2)$ \\
& 0.14232 & $0.96(2)$ & $1.01(2)$ \\
& 0.14280 & $0.95(2)$ & $1.00(2)$ \\
\hline \hline \multirow{4}{*}{0.13600} & 0.13600 & $0.86(1)$ & $0.91(2)$ \\
& 0.14151 & $0.78(1)$ & $0.85(2)$ \\
& 0.14232 & $0.76(2)$ & $0.85(3)$ \\
& 0.14280 & $0.76(1)$ & $0.85(3)$ \\
\hline \hline \multirow{3}{*}{0.14151} & 0.14151 & $0.66(2)$ & $0.76(2)$ \\
& 0.14232 & $0.65(2)$ & $0.76(2)$ \\
& 0.14280 & $0.64(2)$ & $0.76(3)$ \\
\hline \hline \multirow{2}{*}{0.14232} & 0.14232 & $0.64(2)$ & $0.76(2)$ \\
& 0.14280 & $0.64(2)$ & $0.75(3)$ \\
\hline \hline 0.14280 & 0.14280 & $0.63(1)$ & $0.75(3)$ \\
\hline
\end{tabular}

Table 3: Meson masses $M_{H}$ of the pseudoscalar and vector meson at $\beta=6.2$, $T=0$ on a $24^{3} \times 48$ lattice and $T=1.23 T_{c}$ on a $24^{3} \times 8$ lattice. 


\begin{tabular}{|c|c|c|c|}
\hline \multicolumn{4}{|c}{$\mathbf{2 4}^{\mathbf{2}} \times \mathbf{6 4} \times \mathbf{8}, \boldsymbol{\beta}=\mathbf{6 . 4}, \boldsymbol{T}=\mathbf{1 . 6 3} \boldsymbol{T}_{\boldsymbol{c}}$} \\
\hline$\kappa_{1}$ & $\kappa_{2}$ & $M_{P}$ & $M_{V}$ \\
\hline 0.13000 & 0.13000 & $1.055(5)$ & $1.085(2)$ \\
0.14000 & 0.14000 & $0.720(4)$ & $0.756(7)$ \\
0.14030 & 0.14030 & $0.721(4)$ & $0.757(4)$ \\
0.14060 & 0.14060 & $0.719(3)$ & $0.756(3)$ \\
0.14090 & 0.14090 & $0.718(3)$ & $0.755(4)$ \\
\hline
\end{tabular}

Table 4: $\quad$ Meson masses $M_{H}$ of the pseudoscalar and vector meson at $\beta=6.4, T=$ $1.63 T_{c}$ on a $24^{2} \times 64 \times 8$ lattice.

theory, which turns into a linear one at larger $m_{q}$ values. Correspondingly, we chose a fit ansatz of the form

$$
m_{P}=\sqrt{b m_{q}+d m_{q}^{2}}
$$

which returns $b=2.57(1)$ and $d=5.39(6)$, shown as the lowest line in the figure.

Regarding the results at non-vanishing temperature, also at large quark masses we do not observe significant differences to the zero temperature data. However, the meson mass values are again plotted at the zero temperature quark masses, i.e. as a function of $1 / \kappa-1 / \kappa_{c}(T=0)$ with our value of $\kappa_{c}$ at $T=0$. In a fit to the pseudoscalar mass data over the entire $m_{q}$ range, we therefore allow for a non-vanishing chiral limit and replace $m_{q}$ by $m_{q} \rightarrow m_{q}+c$ in the ansatz eq. (5). This fit leads to the second lowest dotted curve in the lower part of Figure 2 with $c=0.005(2), b=2.3(1)$ and $d=5.9(2)$. When fitting the vectormeson over all quark masses we observe a slightly better performance in terms of $\chi^{2}$ of a fit including terms quadratic in $m_{q}, m_{V}=$ $0.413(6)+2.45(5) m_{q}+0.13(5) m_{q}^{2}$, over a linear one, $m_{V}=0.401(5)+2.59(1) m_{q}$. The quadratic term is not large though and the intercepts have their error bars touching each other. Note however that the intercept of the quadratic fit is in slightly better accord with the result of the linear fit to the small quark mass data. The corresponding line in Figure 2 is the quadratic function.

Summarizing the results at $T \simeq 0.93 T_{c}$, we note that the pion (screening) mass exhibits only a very small value in the zero temperature chiral limit which could be absorbed by a slight shift in the critical kappa value. This might also be a finite size effect as the finite temperature lattice is somewhat bigger in spatial volume than the zero temperature ones. A non-vanishing temperature effect is perhaps seen in the vectormeson data which shows an enhancement of 7 to $10 \%$ over the zero temperature data. It remains to be seen, however, whether this finding persists in the continuum limit. On the other hand, our results in the Wilson discretization are in good agreement with observations made in earlier studies using staggered fermions [12]. 

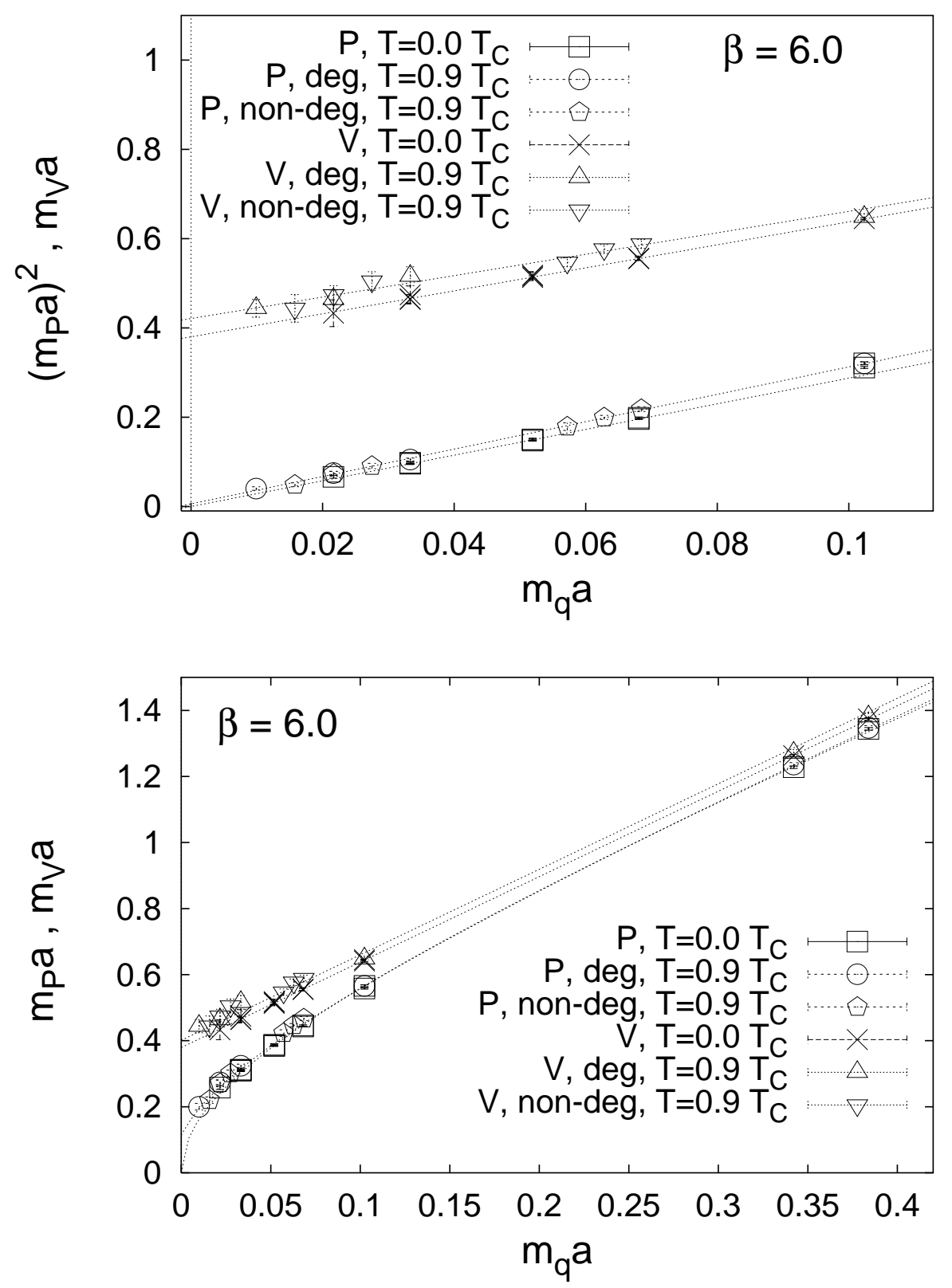

Figure 2: Meson masses at $T \simeq 0.93 T_{c}$ compared with the zero temperature results as a function of the quark mass. The zero-temperature data is partially taken from the literature [33, 34, 39]. The upper plot features the vectormeson mass and the pseudoscalar mass squared in the vicinity of the chiral limit while in the lower part the data for the entire quark mass range are shown. Here both masses are plotted linearly. The lines are fit results explained in the text. 

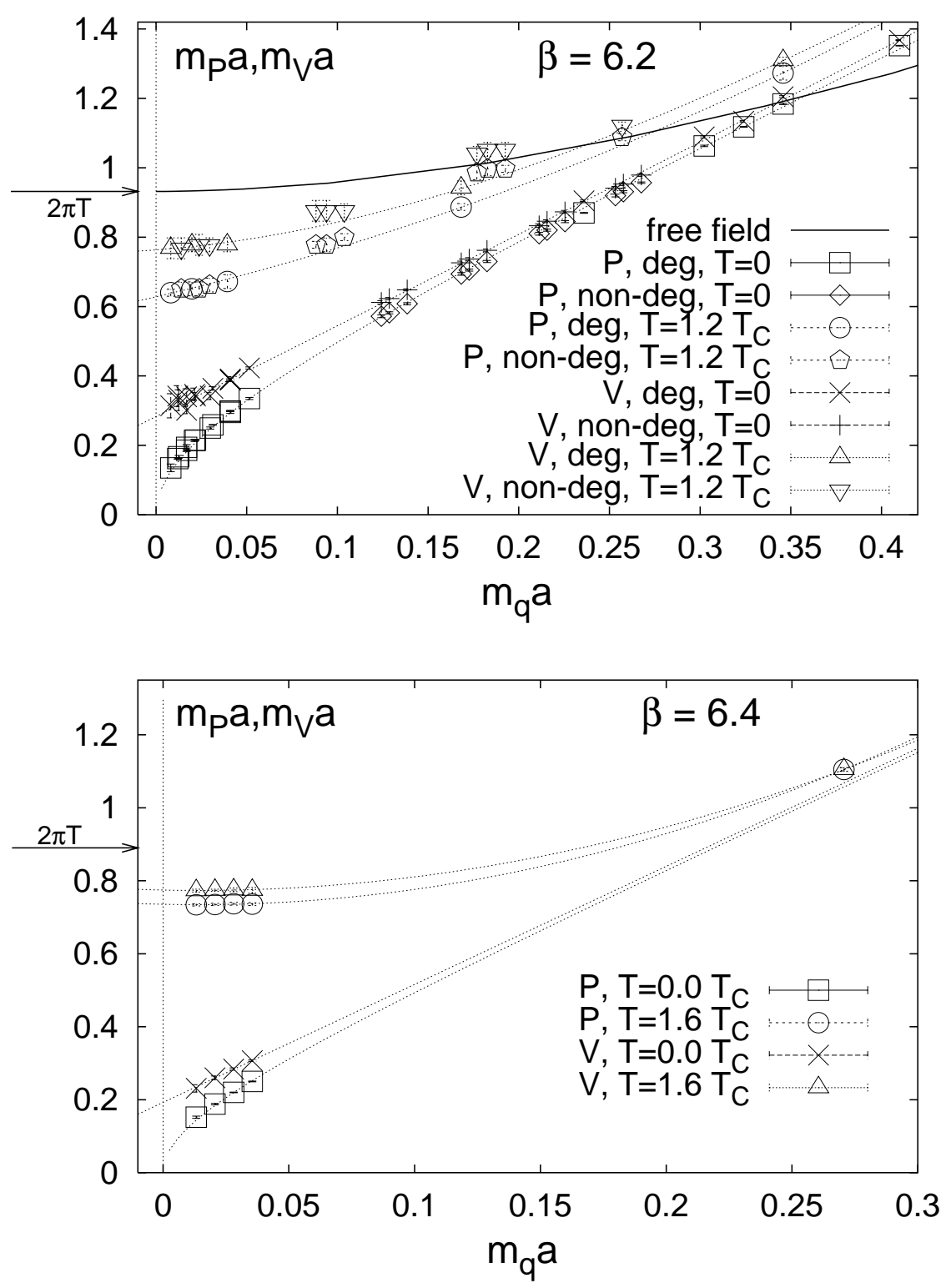

Figure 3: Meson masses at $T \simeq 1.23 T_{c}$ and $1.63 T_{c}$ compared with zero temperature data partially taken from the literature [33, 34, 39].

The results for the meson masses at $\beta=6.2$ and 6.4 corresponding to the two temperatures above $T_{c}$ which are investigated here, $T \simeq 1.23 T_{c}$ and $1.63 T_{c}$ respectively, are summarized in Figure 3. Again, we show $m_{V}$ and $m_{P}$ as a function of the quark mass $m_{q}$ with $\kappa_{c}(T=0)$ determined as explained above from fits to the combined zero temperature pion mass results [34, 35] at small quark mass. The resulting critical $\kappa$ values are $\kappa_{c}=0.14313(2)$ and $0.14141(3)$ at 6.2 and 6.4 , respectively. 
The zero temperature data shows the same behavior as at $\beta=6.0$, including the linearity in $m_{q}$ of the vector meson mass up to the largest quark masses explored. At $\beta=6.2$ we fitted $m_{V}$ at $T=0$ over the entire quark mass range both, quadratically in $m_{q}$, leading to $m_{V}=0.283(2)+2.61(2) m_{q}+0.10(4) m_{q}^{2}$ as well as linearly, $m_{V}=$ $0.279(1)+2.65(5) m_{q}$. Again, the quadratic term was found to be small and the intercepts are stable. Using only data close to the chiral limit leads to an extrapolated value of $m_{\rho}=0.267(8)$, so that the deviation from the fits to the full data set is a little larger than at $\beta=6.0$. The dotted lines given in Figure 3 are the quadratic fits. Note, that the data points labelled by "non-deg" indicate meson masses obtained from propagators with two quarks of non-degenerate masses. They are plotted as a function of an "effective" quark mass $\bar{m}_{q}=\left(m_{q, 1}+m_{q, 2}\right) / 2$ and fit very well on the quadratic curves at $T=0$. The pseudoscalar data was again fitted with the ansatz of eq. (5) which gives $b=1.846(8)$ and $d=6.22(3)$ in this case. This is shown again as the lowest line in the figure.

At $\beta=6.4$ only data at small quark masses were available 34 and we have carried out a linear fit to the vector meson, with the result $m_{V}=0.19(1)+3.23(3) m_{q}$. Although $\kappa_{c}(T=0)$ was obtained from fits to $m_{P}^{2}$ in the vicinity of the chiral limit, in Figure 3 we only show, as lines to guide the eye, the results of fits with eq. (5) under the condition that $m_{P} \lesssim m_{V}$ for large $m_{q}$.

In contrast to the situation at $T<T_{c}$, at both temperatures above the transition point the screening masses are markedly different from the zero temperature results, especially at small quark mass values. The curves interpolating the non-zero temperature data are polynomials of third degree for the vectormeson at $T=1.23 T_{c}$ and of second degree elsewhere. We have chosen this ansatz because above $T_{c}$ no particular functional form is known so far. The curves are the results of fits to data for degenerate quark masses and are mainly meant to guide the eye. The intercepts were obtained from these fits as $m_{\pi}\left(1.23 T_{c}\right)=0.63(2), m_{\rho}\left(1.23 T_{c}\right)=0.74(2)$ and $m_{\pi}\left(1.63 T_{c}\right)=0.735(5), m_{\rho}\left(1.63 T_{c}\right)=0.774(8)$ at the two temperatures respectively. They are discussed in the following.

At high temperatures one expects that the plasma consists of only weakly interacting quarks and gluons. Correspondingly, the correlation functions with mesonic quantum numbers should be described to first approximation by the free propagation of a quark-antiquark pair. In this case the exponential fall-off of the correlation functions is not dominated at large distances by a single mass originating from an isolated pole in the spectral function but rather by all possible energies of quark and antiquark with opposite "momenta" of equal size adding to zero total momentum to which the correlation functions are projected on. Correspondingly, effective masses taken from point to point correlations $(R=0$ in eq. (2)) exhibit a rather marked curvature as function of the separation between the meson operators. Tuning $R$ away from 0 leads to a suppression of higher quark momenta in the momentum sums. This way, it is possible to obtain plateau-like behavior in effective mass plots also in the free quark case. 
The lowest value of quark "momentum" in the temporal direction, i.e. the smallest Matsubara frequency is $\pi T$ due to the antiperiodic boundary condition for fermions in the $t$ direction. This leads to the lowest "energy" contributing to spatial correlation functions of mesonic operators of

$$
m_{H}=2 \sqrt{m_{q}^{2}+(\pi T)^{2}}
$$

in the continuum. On a finite lattice, eq. (6) is subject to finite volume and finite lattice spacing corrections, see e.g. [7]. In order to compare our simulation results at $T>T_{c}$ with the free quark case, we have computed the free quark propagator on a finite lattice analytically and performed the sum over all lattice "momenta" $\overrightarrow{\tilde{p}}$ of the quarks numerically. For definiteness, we then plot the effective mass for point to point correlations at a separation $z=N_{\sigma} / 4$. This results in the solid curve shown in the upper part of Figure 3 .

As in the free case, in our results from the Monte Carlo simulations we are also able to identify plateaux in the effective mass plots when the separation $R$, eq. (21), is tuned. The vectormeson and, to a somewhat less degree, the pseudoscalar masses approach the free quark limit as is seen from Figure 3. The remaining differences are becoming smaller with rising temperature. Moreover, the fact that the masses for nondegenerate quark combinations, plotted at $\bar{m}_{q}=\left(m_{q, 1}+m_{q, 2}\right) / 2$, are above the ones for degenerate quarks would fit into this picture as $2 \sqrt{\bar{m}_{q}^{2}+(\pi T)^{2}}<\sqrt{m_{q, 1}^{2}+(\pi T)^{2}}+$ $\sqrt{m_{q, 2}^{2}+(\pi T)^{2}}$. At large quark masses the Monte Carlo data exceed the free quark curve. This could be explained by noting that in the lattice version of eq. (6) we have used the bare quark mass. At the temperatures investigated one should presumably compare with an effective quark mass which also accounts for a thermal contribution [9]. In addition, it has been argued in the context of dimensional reduction that the (confining) potential of the reduced three-dimensional theory leads to modifications of eq. (6) for screening masses [24]. These modifications will be positive and of order $\sqrt{\sigma_{\text {spat }}}$, where $\sigma_{\text {spat }}$ is the string tension of spatial Wilson loops, and can be estimated to amount to an $\mathcal{O}(10 \%)$ effect [23].

Finally, in Figure 1 our results are compared with data obtained in the staggered

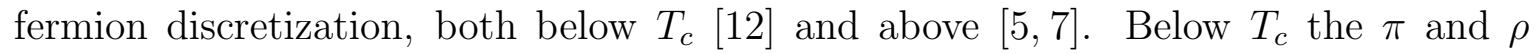
screening masses are shown in the chiral limit. The agreement between the two discretizations is evident. Above $T_{c}$ the staggered data was computed at a small bare quark mass of $m_{q} a=0.02$ while the Wilson results have been extrapolated to the chiral limit. From Figure 3, however, recall that above $T_{c}$ the screening masses are practically independent of $m_{q}$ at small quark masses. Both data sets have been rescaled by the appropriate ratio of $2 \pi$, eq. (6), to its finite lattice size corrected value so that the data can immediately be compared with the continuum expectation for free quarks. Figure 6 shows that above $T_{c}$ the $\rho$ screening mass rapidly approaches $2 \pi$. This holds in both discretizations. The pion however behaves very differently for the two lattice 
actions. In the Wilson case, at $1.63 T_{c}$ the pion screening mass is almost degenerate with the $\rho$ and close to $2 \pi$, with a $\pi$ to $\rho$ ratio of $0.95(1)$. The same approximate degeneracy of $\pi$ and $\rho$ screening masses has been observed in the only other quenched analysis with Wilson quarks available so far [17]. Here a ratio of $0.955(7)$ has been obtained at a temperature of about $1.5 T_{c}$. Quite contrary, the staggered pion screening mass is much smaller than the $\rho, m_{\pi} / m_{\rho}=0.75(2)$ at $1.8 T_{c}$, and even at $5 T_{c}$ reaches only about $75 \%$ of two times the lowest Matsubara frequency. There is no immediate explanation at our hands at least. Possible reasons include the different symmetries of the lattice actions at non-vanishing lattice spacing although the lattice spacings are below $0.1 \mathrm{fm}$, the different aspect ratios although it is hard to see why this should affect the $\pi$ to $\rho$ ratio, and finite volume effects although those would be expected to go the opposite way. Thus, it is hoped that future systematic studies help to resolve this discrepancy between the two lattice fermion formulations.

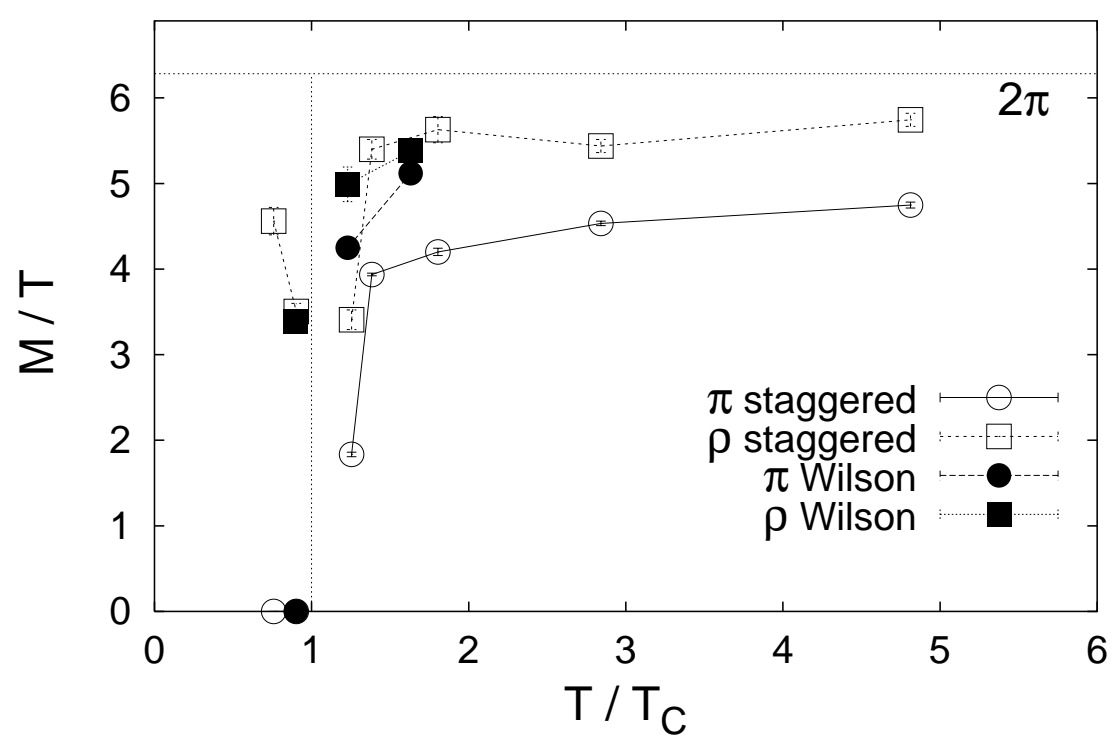

Figure 4: Comparison with the results of quenched staggered simulations below 12 and above $T_{c}$ [5, 7]. The data have been rescaled by the appropriate ratio of $2 \pi$, eq. (6), to its finite lattice size corrected value so that the data can immediately be compared with the continuum expectation for free quarks shown as the horizontal line.

\section{Dispersion relation}

At non-zero temperature, Lorentz invariance is broken because the temporal direction is distinguished as the direction of the four-velocity of the heat bath. As a consequence, unlike the zero temperature case where it depends on the Lorentz invariant scalar $p^{2}$, 
in this case the spectral density will depend on temporal and spatial components $p_{0}$ and $\vec{p}$ separately. At temperatures below the confinement-deconfinement transition the spectrum will still consist of particle excitations, yet, their dispersion relations might be more complicated and reflect the breaking of Lorentzian invariance. The spectral density will be of the form

$$
\rho\left(p_{0}, \vec{p}\right)=2 \pi \epsilon\left(p_{0}\right) \delta\left(p_{0}^{2}-\omega^{2}(\vec{p}, T)\right)
$$

where

$$
\omega^{2}(\vec{p}, T)=m^{2}+\vec{p}^{2}+\Pi(\vec{p}, T)
$$

contains the temperature dependent vacuum polarization tensor $\Pi(\vec{p}, T)$. As a simple example, assume that the temperature effects can be absorbed into a temperature dependent mass $m(T)$ and a coefficient $A(T)$ which might also be temperature dependent and different from 1 ,

$$
\omega^{2}(\vec{p}, T) \simeq m^{2}(T)+A^{2}(T) \vec{p}^{2}
$$

Such an approximation might hold at least at small temperatures. In this case, at zero momentum the temporal correlator will decay with the so-called pole mass $m(T)$

$$
C(\vec{p}=0, t) \sim \exp (-m(T) t)
$$

whereas the spatial correlation function has an exponential fall-off

$$
C(\overrightarrow{\tilde{p}}=0, z) \sim \exp \left(-m_{\mathrm{sc}}(T) z\right)
$$

determined by the screening mass $m_{\mathrm{sc}}(T)=m(T) / A(T)$ which differs from the pole mass if $A(T) \neq 1$. At non-vanishing "momentum", $\overrightarrow{\tilde{p}} \neq 0$, the exponential decrease of the spatial correlator is described by $\omega_{\mathrm{sc}}$,

$$
C(\overrightarrow{\tilde{p}}, z) \sim \exp \left(-\omega_{\mathrm{sc}} z\right)
$$

where in this particularly simple example $\omega_{\text {sc }}$ is given as

$$
\omega_{\mathrm{sc}}^{2}=\vec{p}_{\perp}^{2}+\frac{\omega_{n}^{2}}{A^{2}}+m_{\mathrm{sc}}^{2}
$$

Comparing different projections to Matsubara frequencies $\omega_{n}=2 \pi T n$ and to spatial momenta $\vec{p}_{\perp}$ might thus reveal a dispersion relation different from the zero temperature one and, moreover, indicate a coefficient $A$ different from 1 and correspondingly a difference between pole and screening mass.

The formulae given above are the continuum dispersion relations. They are modified on the lattice. Zero temperature studies [34, 38] have shown that a lattice dispersion of the form

$$
\sinh ^{2}\left(\frac{E}{2}\right)=\sum_{k} \sin ^{2}\left(\frac{p_{k}}{2}\right)+\sinh ^{2}\left(\frac{M}{2}\right)
$$


arising from an effective boson action with a nearest-neighbor kinetic term, is best capable to describe lattice data at non-zero momentum.

We investigated the lattice dispersion relation of mesons below $T_{c}, T \simeq 0.93 T_{c}$, for different quark masses and compared it with $T=0$ data. In Figure 5 we show our results obtained in the pseudoscalar channel at $\kappa=0.141$. We have plotted $M_{\pi} a$ obtained by subtracting the momentum contribution $\sin ^{2}\left(p_{k} a / 2\right)$ from both sides of eq. (14) where $E$ is the fitted coefficient of the exponential fall-off in $z$ of the spatial correlation function. Results at other $\kappa$ values investigated are very similar.

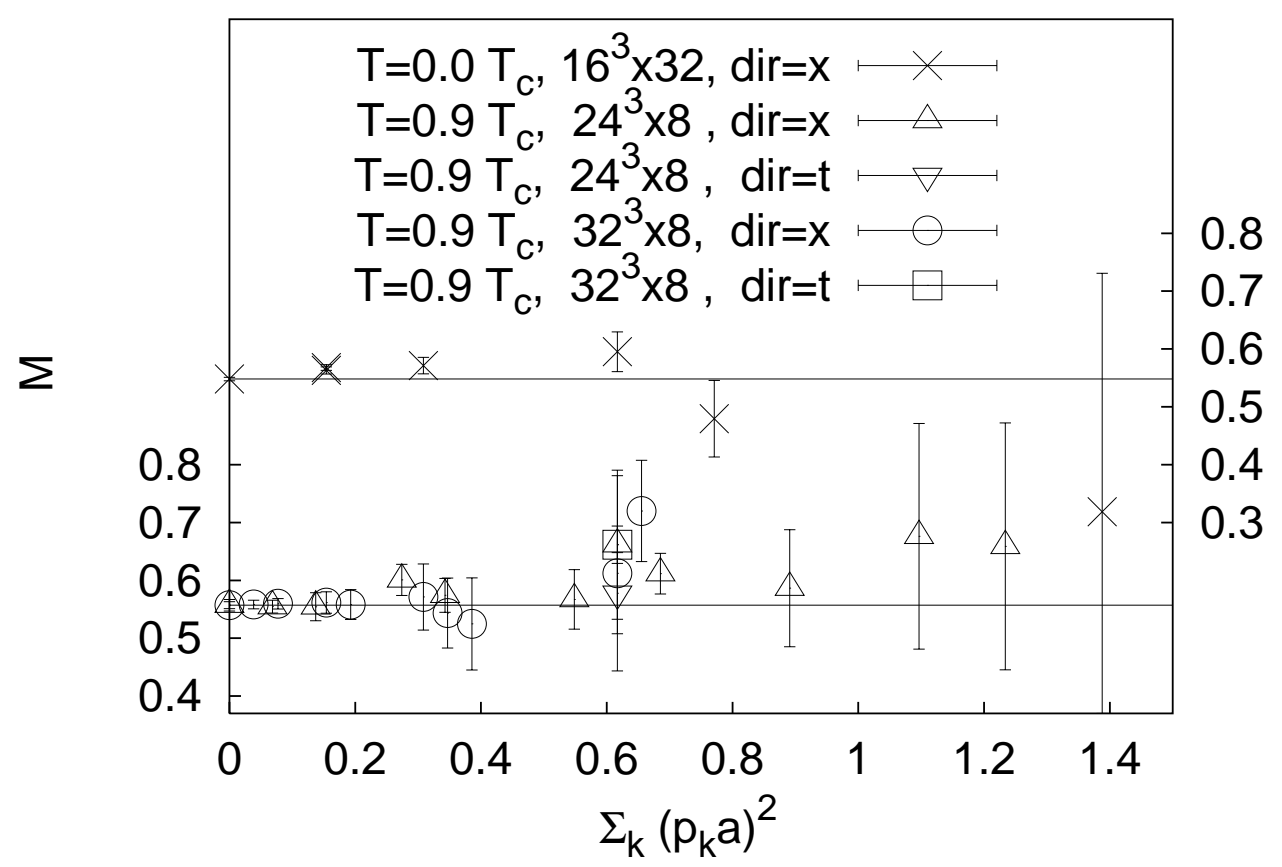

Figure 5: The lattice dispersion relation shown for the pseudoscalar channel at $\kappa=0.141$. In the figure, "dir" indicates whether spatial or temporal components of $\left(p_{x}, p_{y}, p_{t}\right)$ were chosen to be different from 0 . The horizontal lines denote the dispersion relation eq. (14). Note that the $T=0$ data has been shifted upwards, right scale.

In Figure 5, a $p_{k}$ independence of the data indicates that eq. (14) is the correct dispersion relation. Indeed, it seems to be favored by the zero temperature data. As in [38, using a different dispersion relation led to data points rising or falling with $p_{k}$.

Regarding the data at $T=0.93 T_{c}$, again we observe that the zero-temperature dispersion, eq. (14), is describing the data for non-vanishing spatial momentum components properly. Note that the figure contains data from two different lattice sizes, leading to different values for the spatial momenta. Moreover, the single data point for 
the lowest bosonic Matsubara frequency $\omega_{1}=2 \pi T$ is also lying on the horizontal line, suggesting that the coefficient $A$ of eq. (13) is not too far from unity. Of course, this conclusion is tied to the applicability of eq. (13), moreover, the statistical significance is certainly not overwhelming. Nevertheless, this result might be taken as further support for the difference between pole and screening masses not being too large at $T \simeq 0.93 T_{c}$, see also [17].

In addition to the investigation below $T_{c}$, we also computed the dispersion relation in mesonic channels in the deconfined phase. The results are shown and compared with the free quark case in Figure 6. The former have been determined by fits in the same way as the masses while for the latter, to be definite we use the effective energies at $N_{\sigma} / 4$, obtained from sink operators with $R=4$. Both, Monte Carlo as well as free quark results are somewhat dependent on the quark-antiquark distance. As can be read off the figure, at $1.23 T_{c}$ the data for spatial momenta show systematic deviations from the preferred zero-temperature dispersion relation. These differences have almost disappeared at $1.63 T_{c}$ and the data are becoming consistent with the zerotemperature dispersion relation which also happens to describe the free quark case. For the lowest temporal mesonic "momentum", $p_{t}=2 \pi T$, at $1.23 T_{c}$ pseudoscalar and vector "energy" both are systematically lower than the corresponding results for a spatial momentum of exactly the same value showing that Lorentzian symmetry is disturbed. At $1.63 T_{c}$ the data follow the free quark behavior, in particular insofar in the vector channel the "energy" at $p_{t}=2 \pi T$ is much smaller than the "energy" at the same spatial momentum周.

\section{Wave function}

Bethe-Salpeter amplitudes provide information about the probability of finding a prearranged configuration of quarks inside a hadron. Their general definition is given by

$$
\Phi(\vec{R})=\langle 0|\mathcal{O}(\vec{R})| H(\vec{p})\rangle
$$

where, for $|H\rangle$ being a meson state, the operator $\mathcal{O}(\vec{R})$ annihilates a quark-antiquark pair with the appropriate quantum numbers separated by $\vec{R}$. The choice of the operator is not unique. On the lattice, Bethe-Salpeter amplitudes have been studied in Coulomb and in Landau gauge or by using various gauge-invariant definitions [36, 40 43]. The various methods treat the gluon flux tube connecting quark and antiquark in a different way so that it may not be surprising that different definitions of the amplitudes have lead to different results (see however [43]). Moreover, an

\footnotetext{
${ }^{a}$ Note that this, at first sight strange behavior can easily be reproduced in the continuum if only the lowest quark Matsubara frequency would contribute to the correlation function. Of course, in this case pseudoscalar and vector channel would lead to the same result. Apparently, the interplay between higher quark momenta and the spin structure is important.
} 

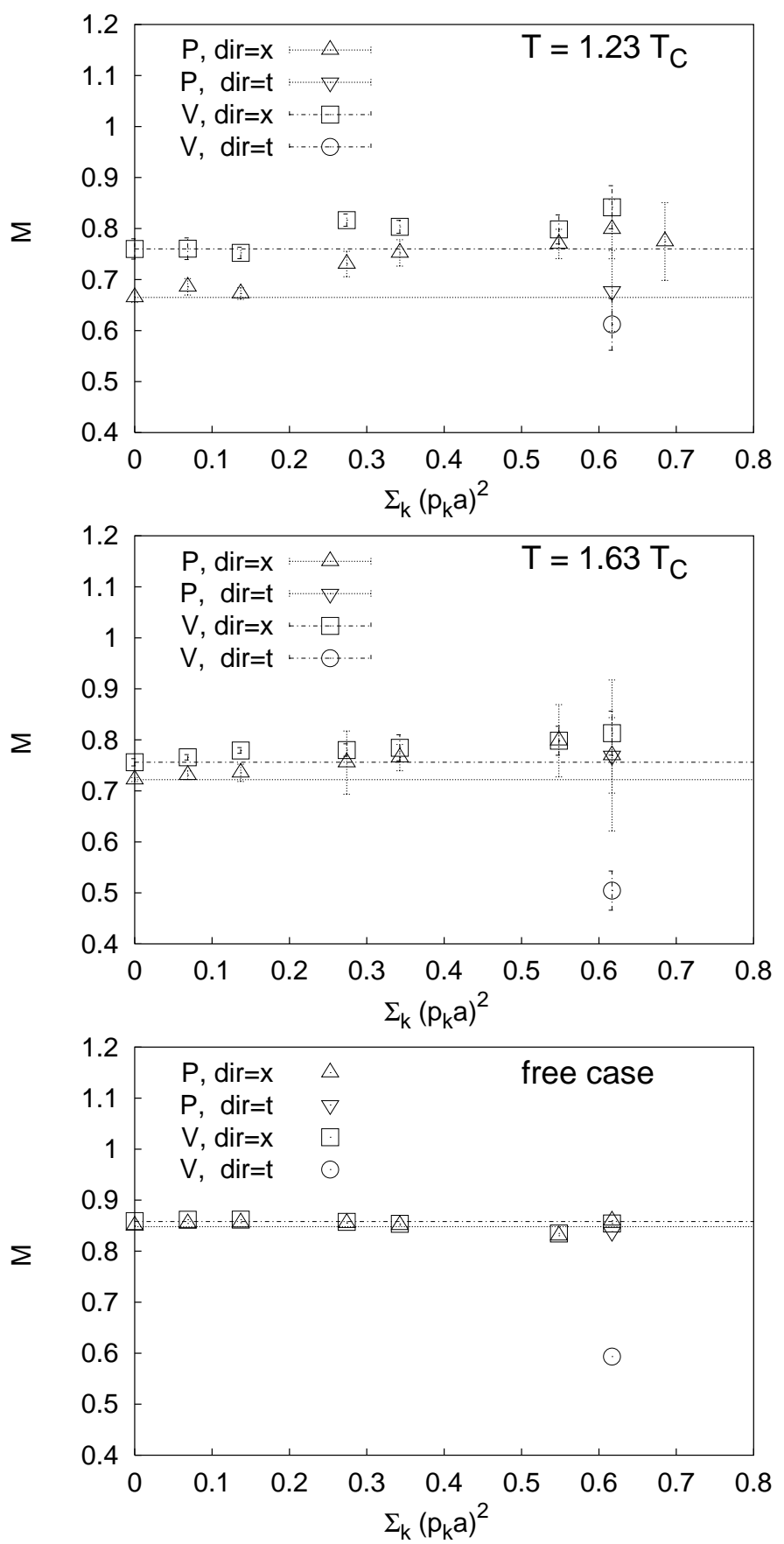

Figure 6: The lattice dispersion relation at two temperatures above $T_{c}$ and in the free quark case. Shown are both the results for the pseudoscalar and the vector channel. The bare quark mass is roughly equal to $m_{s}$. Again, "dir" indicates whether spatial or temporal components of $\left(p_{x}, p_{y}, p_{t}\right)$ were chosen to be different from 0 . The horizontal lines denote the dispersion relation eq. (14). 
immediate connection between the size of a hadron as extracted from the amplitudes and the measured radii has not been demonstrated. Nevertheless, the Bethe-Salpeter amplitudes do provide some qualitative insight into the quark distribution inside a hadron, and in particular they should be able to capture differences in the structure of hadronic excitations at zero and high temperature if these are present [19.

As a by-product of the attempt to obtain a better projection onto the ground state, in this paper we use a gauge-invariant definition of $\mathcal{O}(\vec{R})$ as given in eq. (2) [36]. We analyze S-wave states only as this operator is invariant under $90^{\circ}$ rotations. The Bethe-Salpeter amplitudes are determined from simultaneous two-state fits to all the correlation functions $C_{R}$ at all $R$ values at which they were calculated. In these fits the ground state mass was required to be the same at all $R$ whereas the mass of the second state as well as the amplitudes for both ground and excited state were allowed to be $R$ dependent. Again, the fit interval was varied and we checked for stability of the fit results under this variation as well as for agreement of the ground state mass with the numbers obtained as explained in section 3 .

The procedure proved stable enough to extract the Bethe-Salpeter amplitude for the ground state as well as to obtain an estimate for the excited state. An example of the results is shown in Figure 7 . Here, the wave functions have been normalized to 1 at $R=0$. Note that the amplitude of the excited state is vanishing at about $1 / 2 \mathrm{fm}$. This corresponds with the observation that at large $R$ effective masses approach the plateaux in effective mass plots from below, see Figure 1. The results for the excited states are more sensitive to variations of the fit interval since at small separations from the source more than one excited state can contribute whereas at large separations the excited state dies out. We will thus concentrate on the ground state results in the following.

The ground state wave functions 1 are fitted with slightly modified hydrogen S-wave functions 42 ,

$$
\Phi(R) \propto \exp \left(-\left(R / a_{0}\right)^{\nu}\right)
$$

with the free parameters $a_{0}$ and $\nu$. In non-relativistic potential models the power of $R$ is obtained as $\nu=1$ for a purely Coulombic and $\nu=3 / 2$ for a linear potential. The wave functions can be used to compute an estimate of the size of the meson [42],

$$
\left\langle r^{2}\right\rangle=\int d^{3} \vec{R}\left(\frac{R}{2}\right)^{2} \Phi^{2}(R) / \int d^{3} \vec{R} \Phi^{2}(R)=\frac{a_{0}^{2} \Gamma(5 / \nu)}{4^{1+1 / \nu} \Gamma(3 / \nu)} .
$$

The results of these fits to the pseudoscalar as well as vector meson ground state are summarized in Table 5. We first want to stress that the measured zero temperature wave functions, i.e. the parameter $a_{0}$ and the radius respectively, scale with $\beta$ as the

\footnotetext{
${ }^{\mathrm{b}}$ The excited states could be well fitted by the ansatz $\Phi(R) \propto\left(1-R / b_{1}\right) \exp \left(-\left(R / a_{1}\right)^{\nu}\right)$ which accounts for the node in the wave function.
} 


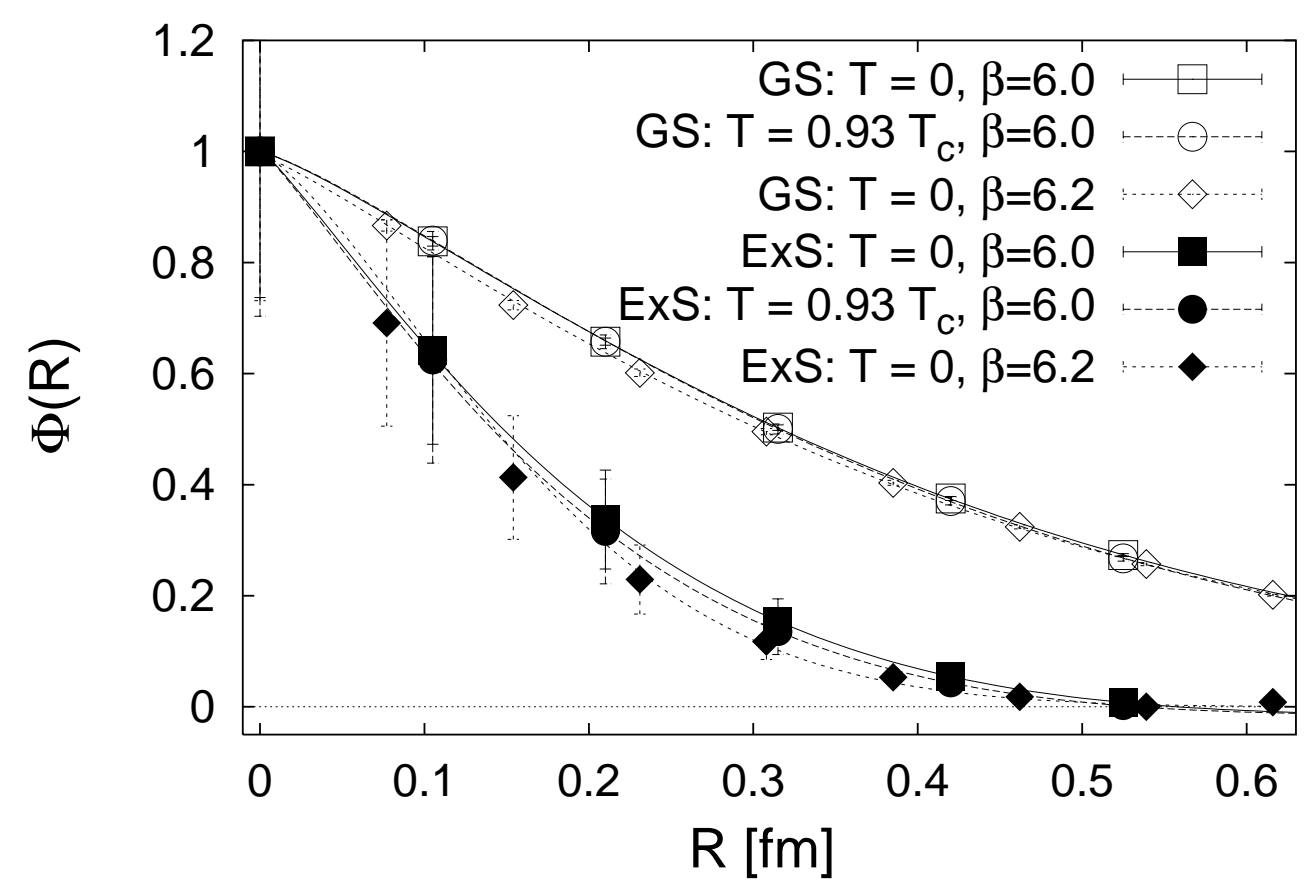

Figure 7: Wave functions for the ground (GS) and excited state (ExS) of the pseudoscalar at $T=0$ and $T=0.93 T_{c}$, for $m_{q} \approx m_{s}$

lattice spacing when the (physical) quark mass is fixed. This is lending further support that the investigated observable is physical.

At $T=0$, the obtained values for the parameter $\nu$ lie between the values for a purely Coulombic and a purely linear potential. The values for $\nu$ are slightly increasing with the quark mass and they are larger for the vector meson than for the pseudoscalar. Moreover, the values for $a_{0}$ and for the radii are also increasing with the quark mass and again are larger for the vector meson than for the pseudoscalar. Both effects are consistent with the experimental observation that the $\rho$ meson is larger than the pion and thus probes the linear rising part of the potential at larger distances. Compared to the (Coulomb gauge) results of 42] at the same pseudoscalar to vector meson mass ratio, we observe perfect agreement for the parameter $\nu$ of the pseudoscalar ground state whereas our $\nu$ value for the vector meson is slightly smaller. The second moments of the meson wave functions, eq. (17), are about $10 \%$ larger than in 42. Still, as reported there, we obtain radii which are about a factor two smaller than sizes obtained from form factor measurements. Whether this discrepancy is due to the quenched approximation i.e. to not taking proper account of a pion cloud still has to be speculated at the present stage.

At $T=0.93 T_{c}$ the pseudoscalar as well as vector meson (spatial) wave functions 


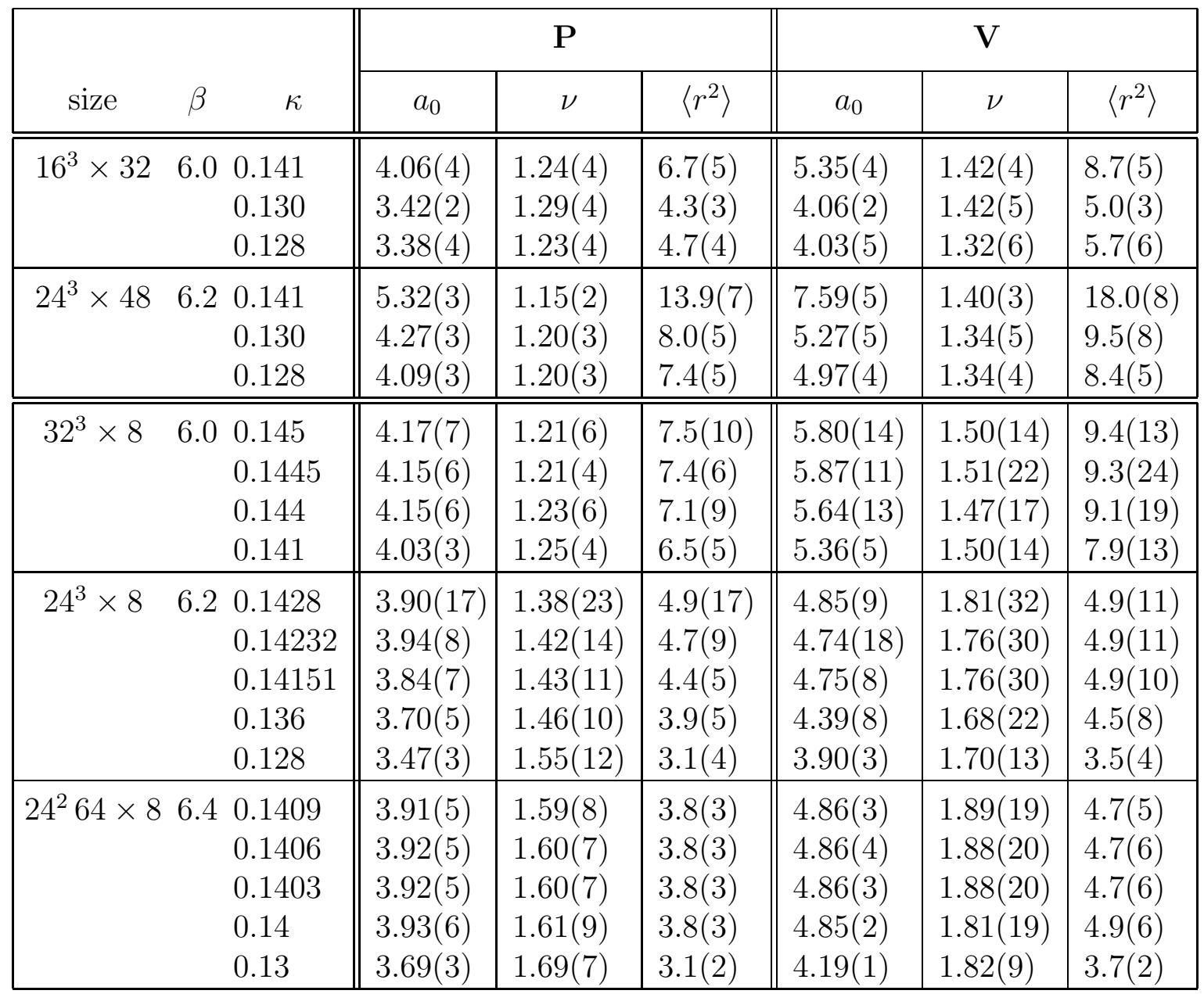

Table 5: Results for the coefficients $a$ and $\nu$ of the ground state wave function, eq. (16), as well as for the mean radius squared, eq. (17), for the pseudoscalar (P) and vector meson $(\mathrm{V})$.

agree with the (temporal) zero temperature ones at quark masses where we have data for both. This also holds for the excited state, see Figure 0. At this temperature we also have data at small quark masses so that we can attempt a chiral extrapolation. A fit to the squared radii which is linear in $m_{P S}^{2}$ leads to values of $\left\langle(r / a)_{\pi}^{2}\right\rangle=7.6(6)$ and $\left\langle(r / a)_{\rho}^{2}\right\rangle=9.6(12)$, respectively, which are in agreement within errors with the zero-temperature numbers reported in [42].

At the two temperatures above $T_{c}$ it is observed that the spatial wave functions in lattice units practically remain unchanged between the two $\beta$ values. This is distinctively different from the zero temperature situation.

In more detail, fits to the wave functions with eq. (16) return the values given in Table 5. The results for the parameter $a_{0}$ are almost independent of $\beta$. The parameter 


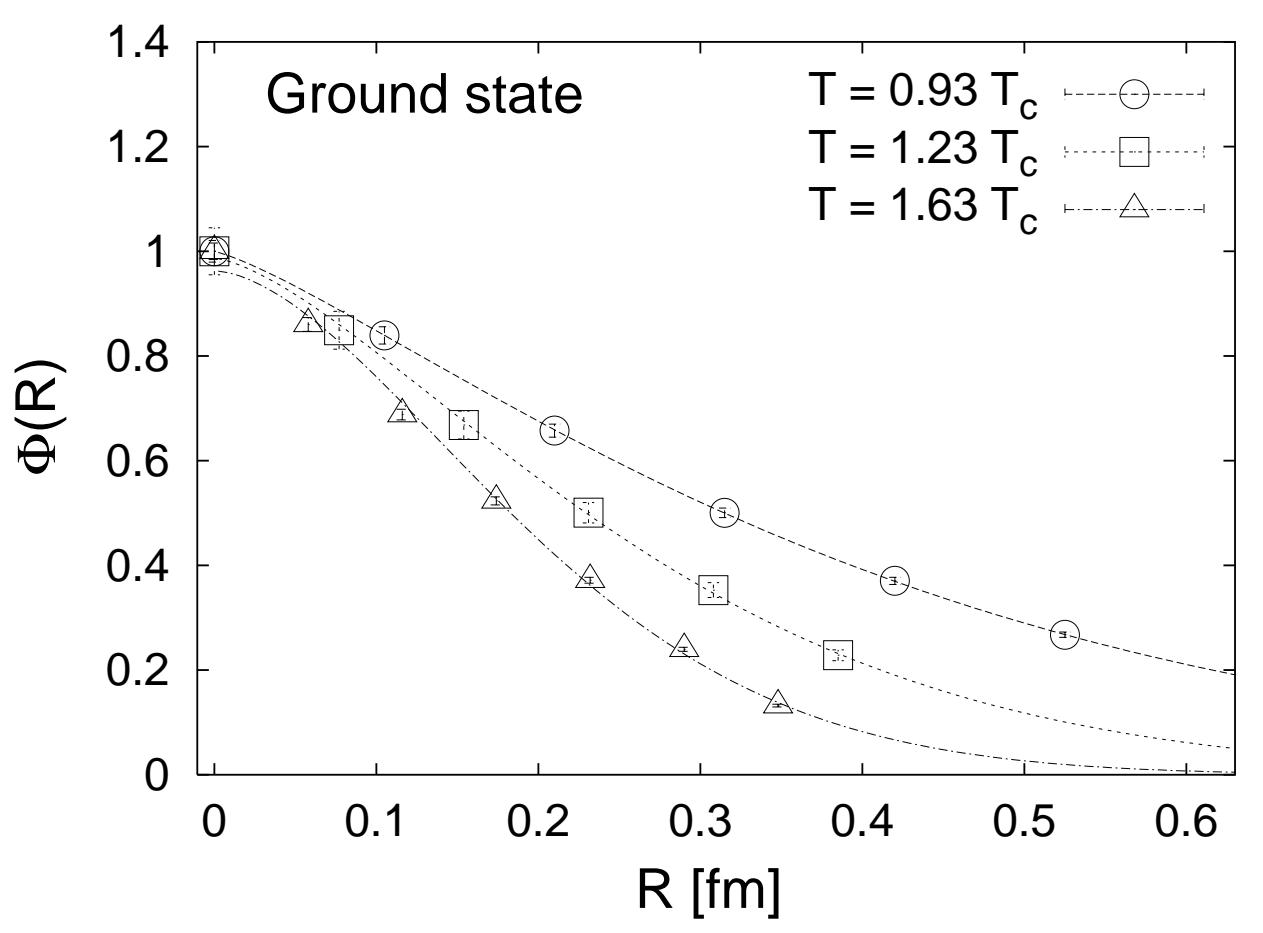

Figure 8: Wave functions for the ground state of the pseudoscalar at $T \neq 0$, for $m_{q} \approx m_{s}$

$\nu$ takes on values which are larger than at zero temperature. Close to the chiral limit, $\nu$ changes from about 1.4 at $1.23 T_{c}$ to 1.6 at $1.63 T_{c}$ for the pion while for the $\rho$ the change with temperature is smaller, $\nu \simeq 1.8$ and 1.9 respectively. The second moment of the wave function, $\left\langle r^{2}\right\rangle$, in lattice units stays constant within errors for the $\rho$ while for the pion with rising temperature we observe a decrease of the central values, with error bars just overlapping. As the quark mass is increased the size of the state is slightly shrinking.

Translated to physical distances a shrinking of the states' size with temperature is clearly oberved, Figure 8. At least qualitatively, this observation is in accord with the anticipated behavior [20,24] of

$$
\Phi(R) \propto \exp \left(-\sqrt{\sigma_{\text {spat }}(T) \pi T} R^{3 / 2}\right)
$$

where $\sigma_{\text {spat }}(T)$ is the temperature dependent string tension of spatial Wilson loops, $\sqrt{\sigma_{\text {spat }}(T)}=c g^{2}(T) T$. Given the small variation of the effective coupling constant $g(T)$ between $T=1.23$ and $1.63 T_{c},\left\langle r^{2}\right\rangle$ should thus shrink proportional to $1 / T^{2}$ to first approximation. Indeed, this seems to hold for the vector meson whereas the variation with temperature of the pion radius is stronger. Moreover, the power of the $R$ dependence is not quite as anticipated. A more detailed investigation along the lines of [20] is planned for the future. 


\section{Conclusion}

In this paper we have presented results of a lattice investigation of meson correlation functions built from improved Wilson quarks on quenched gauge field configurations. Below the confinement-deconfinement transition temperature we do not observe any significant difference between finite temperature screening masses and zero temperature masses. This agrees with earlier findings obtained in the staggered discretization. Likewise, the spatial wavefunctions at non-vanishing temperature agree with wave functions at $T=0$. Moreover, the dispersion relation does not seem to depend on whether the "momenta" are chosen to point in the spatial or temporal direction. This can be viewed as a hint towards the similarity of screening and pole masses as has also been reported in an earlier study on anisotropic lattices. Above the critical temperature, we have computed meson screening masses which in both cases, pseudoscalar as well as vector channel, are close to twice the lowest quark Matsubara frequency. Moreover, at $1.63 T_{c}$ the "momentum" dependencies of the correlation functions are in agreement with the free quark case while at $1.23 T_{c}$ the situation certainly is more complex. Finally, the spatial wave functions, at least qualitatively, behave in accord with the expectation that the temperature dependence of the spatial string tension leads to a narrowing of the wave functions with rising temperature. We thus come to conclude that above $T_{c}$ the spatial correlation functions are consistent with a leading two quark contribution. This still leaves room for infrared non-perturbative effects. Note in this respect that HTL-resummed perturbation theory does not show significant changes in (temporal) correlation functions although the underlying spectral function is quite different from the free case [44]. It is our hope that more refined analysis techniques along the lines set in 45] and perhaps more systematic studies of infrared properties on large lattices extend the knowledge about hadronic excitations in the plasma phase.

\section{Acknowledgement}

This work was supported by the TMR network ERBFMRX-CT-970122 and the DFG grant Ka 1198/4-1. The numerical work has been carried out on the CRAY T3E at the Konrad Zuse Institut Berlin and to a small part on the CRAY T3E at NIC, FZ Jülich. We thank both institutions for allocation of computer time and H. Stüben as well as N. Attig for their generous help.

\section{References}

[1] G. Akakichiev et al. (CERES Collaboration), Phys. Lett. B422 (1998) 405. 
[2] R. Rapp and J. Wambach, "Chiral Symmetry Restoration and Dileptons in Relativistic Heavy-Ion Collisions", hep-ph/9909229.

[3] C. DeTar, Phys. Rev. D32 (1985) 276; Phys. Rev. D37 (1987) 2328.

[4] C. DeTar and J. B. Kogut, Phys. Rev. Lett. 59 (1987) 399.

[5] A. Gocksch, P. Rossi and U.M. Heller, Phys. Lett. B205 (88) 334.

[6] S. Gottlieb et al., Phys. Rev. Lett. 59 (1987) 1881.

[7] K. D. Born et al., Phys. Rev. Lett. 67 (1991) 302.

[8] S. Gupta, Phys. Lett. B288 (1991) 171.

[9] G. Boyd, S. Gupta and F. Karsch, Nucl. Phys. B385 (1992) 482.

[10] C. Bernard et al., Phys. Rev. D45 (1992) 3854.

[11] G. Boyd et al., Z. Phys. C64 (1994) 331.

[12] G. Boyd et al., Phys. Lett. B349 (1995) 170.

[13] S. Gottlieb et al., Phys. Rev. D47 (1993) 3619, Phys. Rev. D55 (1997) 6852.

[14] J.B. Kogut, J.-F. Lagaë and D.K. Sinclair, Phys. Rev. D58 (1998) 54504.

[15] T. Hashimoto, T. Nakamura and I. O. Stamatescu, Nucl. Phys. B400 (1993) 267.

[16] T. Hashimoto, T. Nakamura and I. O. Stamatescu, Nucl. Phys. B406 (1993) 325.

[17] Ph. de Forcrand et al. (QCD-TARO), Phys. Rev. D63 (2001) 054501.

[18] P. Vranas (QCDSP Coll.), Nucl. Phys. Proc. Suppl. 83 (2000) 414.

[19] C. Bernard et al., Phys. Rev. Lett. 68 (1992) 2125.

[20] V. Koch et al., Phys. Rev. D46 (1992) 3169.

[21] C. Borgs, Nucl. Phys. B261 (1985) 455.

[22] E. Manousakis and J. Polonyi, Phys. Rev. Lett. 58 (1987) 847.

[23] F. Karsch, E. Laermann and M. Lütgemeier, Phys. Lett. B346 (1995) 94.

[24] T.H. Hansson and I. Zahed, Nucl. Phys. B374 (1992) 277.

[25] B. Sheikholeslami and R. Wohlert, Nucl. Phys. B259 (1985) 572.

[26] N. Cabibbo and E. Marinari, Phys. Lett. 119B (1982) 387. 
[27] K. Fabricius and O. Haan, Phys. Lett. 143B (1984) 459;

A. Kennedy and B. Pendleton, Phys. Lett. 156B (1985) 393.

[28] S. Adler, Phys. Rev. D23 (1981) 2901.

[29] R.G. Edwards, U.M. Heller and T.R. Klassen, Nucl. Phys. B517 (1998) 377 and references therein.

[30] B. Beinlich et al., Eur. Phys. J. C6 (1999) 133.

[31] C.C. Paige and M.A. Saunders, J. Num. Anal. 12 (1975) 617.

[32] A. Frommer et al., Int. J. Mod. Phys. C5 (1994) 1073 and references therein.

[33] UKQCD Coll., Phys. Rev. D49 (1994) 1594.

[34] C. Allton et al., Nucl. Phys. B489 (1997) 427.

[35] UKQCD Coll., Phys. Rev. D49 (1994) 474.

[36] UKQCD Coll., Phys. Rev. D51 (1995) 6403.

[37] M. Albanese et al., Phys. Lett. 192B (1987) 163.

[38] T. Bhattacharya et al., Phys. Rev. D53 (1996) 6486.

[39] C. Allton et al. and APE Coll., Nucl. Phys. B413 (1994) 461.

[40] B. Velikson and D. Weingarten, Nucl. Phys. B249 (1985) 433.

[41] M.-C. Chu, M. Lissia and J. Negele, Nucl. Phys. B360 (1991) 31.

[42] M.W. Hecht and T.A. DeGrand, Phys. Rev. D46 (1992) 2155

[43] R. Gupta, D. Daniel and J. Grandy, Phys. Rev. D48 (1993) 3330.

[44] F. Karsch, M.G. Mustafa and M.H. Thoma, Phys. Lett. B497 (2001) 249.

[45] M. Asakawa, T. Hatsuda and Y. Nakahara, hep-lat/0011040;

I. Wetzorke and F. Karsch, hep-lat/0008008. 